\title{
ARTICLE
}

\section{Lactation is associated with altered metabolomic signatures in women with gestational diabetes}

\author{
Daniela Much ${ }^{1,2,3,4}$ - Andreas Beyerlein ${ }^{1,4}$ - Alida Kindt ${ }^{4,5}$ - Jan Krumsiek ${ }^{4,5}$ • \\ Ferdinand Stückler ${ }^{4,5}$ - Michaela Rossbauer ${ }^{1,2,3}$ • Anna Hofelich ${ }^{1,4}$. \\ David Wiesenäcker ${ }^{1,2,6}$ - Susanne Hivner ${ }^{1,2,3,4}$ - Melanie Herbst ${ }^{1,2,3,4}$. \\ Werner Römisch-Margl $^{4,7} \cdot$ Cornelia Prehn $^{8}$ • Jerzy Adamski ${ }^{4,8,9}$.

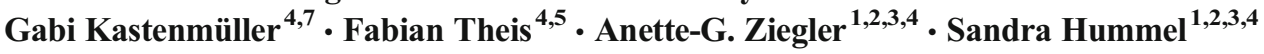

Received: 18 April 2016 / Accepted: 20 June 2016 / Published online: 16 July 2016

(C) Springer-Verlag Berlin Heidelberg 2016

\begin{abstract}
Aims/hypothesis Lactation for $>3$ months in women with gestational diabetes is associated with a reduced risk of type 2 diabetes that persists for up to 15 years postpartum. However, the underlying mechanisms are unknown. We examined whether in women with gestational diabetes lactation for $>3$ months is associated with altered metabolomic signatures postpartum.

Methods We enrolled 197 women with gestational diabetes at a median of 3.6 years (interquartile range $0.7-6.5$ years) after delivery. Targeted metabolomics profiles (including 156 metabolites) were obtained during a glucose challenge test. Comparisons of metabolite concentrations and ratios between women who lactated for $>3$ months and women who lactated for $\leq 3$ months or not at all were performed using linear regression with adjustment for age and BMI at the postpartum visit, time since delivery, and maternal education level, and correction for multiple testing. Gaussian graphical modelling was used to generate metabolite networks.
\end{abstract}

Electronic supplementary material The online version of this article (doi:10.1007/s00125-016-4055-8) contains peer-reviewed but unedited supplementary material, which is available to authorised users.

Sandra Hummel

sandra.hummel@helmholtz-muenchen.de

1 Institute of Diabetes Research, Helmholtz Zentrum München, Ingolstaedter Landstr. 1, 85764 Neuherberg, Germany

2 Forschergruppe Diabetes, Klinikum rechts der Isar, Technische Universität München, Munich, Germany

3 Forschergruppe Diabetes e.V., Neuherberg, Germany

4 German Center for Diabetes Research (DZD), Munich-Neuherberg, Germany
Results Lactation for $>3$ months was associated with a higher total lysophosphatidylcholine/total phosphatidylcholine ratio; in women with short-term follow-up, it was also associated with lower leucine concentrations and a lower total branchedchain amino acid concentration. Gaussian graphical modelling identified subgroups of closely linked metabolites within phosphatidylcholines and branched-chain amino acids that were affected by lactation for $>3$ months and have been linked to the pathophysiology of type 2 diabetes in previous studies.

Conclusions/interpretation Lactation for $>3$ months in women with gestational diabetes is associated with changes in the metabolomics profile that have been linked to the early pathogenesis of type 2 diabetes.

Keywords Gestational diabetes $\cdot$ Lactation $\cdot$ Metabolic networks $\cdot$ Metabolomic profile $\cdot$ Type 2 diabetes

5 Institute of Computational Biology, Helmholtz Zentrum München, Neuherberg, Germany

6 Department of Pediatrics, Kinderklinik München Schwabing, Klinikum rechts der Isar, Technische Universität München, Munich, Germany

7 Institute of Bioinformatics and Systems Biology, Helmholtz Zentrum München, Neuherberg, Germany

8 Genome Analysis Center, Institute of Experimental Genetics, Helmholtz Zentrum München, Neuherberg, Germany

9 Lehrstuhl für Experimentelle Genetik, Technische Universität München, Freising-Weihenstephan, Germany 


\begin{tabular}{|c|c|}
\hline \multicolumn{2}{|c|}{ Abbreviations } \\
\hline BCAA & Branched-chain amino acids \\
\hline ESI & Electrospray ionisation \\
\hline FIA-MS/MS & $\begin{array}{l}\text { Flow injection analysis tandem mass } \\
\text { spectrometry }\end{array}$ \\
\hline GGM & Gaussian graphical model \\
\hline ISI & Insulin sensitivity index \\
\hline IQR & Interquartile range \\
\hline LC-MS/MS & $\begin{array}{l}\text { Liquid chromatography tandem mass } \\
\text { spectrometry }\end{array}$ \\
\hline Lyso-PC & Acyl-lysophosphatidylcholine \\
\hline $\mathrm{PC}$ & Phosphatidylcholine \\
\hline PINGUIN & $\begin{array}{l}\text { Postpartum Intervention in Women } \\
\text { with Gestational Diabetes Using Insulin }\end{array}$ \\
\hline POGO & $\begin{array}{l}\text { Postpartum Outcomes in Women with } \\
\text { Gestational Diabetes and their Offspring }\end{array}$ \\
\hline
\end{tabular}

\section{Introduction}

The beneficial effects of lactation are widely considered to be positive health outcomes in the child $[1,2]$. However, there is increasing evidence to suggest that lactation also has longterm health benefits for the mother. We recently reported that lactation in women with gestational diabetes was associated with a considerable long-term reduction in the risk of developing type 2 diabetes postpartum [3]. This risk reduction was most pronounced in women who lactated for $>3$ months, and the beneficial effect of lactation on the risk of diabetes was apparent for up to 15 years postpartum [3]. However, the mechanisms that may explain this protective association are unclear [4].

A high pre-pregnancy BMI and extreme weight gain during pregnancy were associated with both a shorter duration of lactation and an increased risk of type 2 diabetes postpartum $[5,6]$. A lactating mother transfers $1674-2510 \mathrm{~kJ}(400$ $600 \mathrm{kcal})$ per day to her infant, which may facilitate postpartum weight loss compared with non-lactating mothers. Consequently, it was hypothesised that the association between duration of lactation and maternal type 2 diabetes risk may be confounded by maternal BMI $[7,8]$. However, in our previous analysis [3] and in studies of healthy women [9, 10], adjusting for BMI had no impact or a very small impact on the association between lactation and type 2 diabetes risk. Moreover, a recent population-based study demonstrated that healthy lifestyle behaviours, which may be more prevalent in lactating mothers, did not confound the association between lactation and type 2 diabetes risk [10].

Several studies conducted during or shortly after lactation have revealed that fasting blood glucose, postprandial blood glucose and insulin concentrations were lower [11, 12], atherogenic blood lipid concentrations were lower [13] and insulin sensitivity was improved [11] in healthy, middle-aged lactating women compared with non-lactating women. These short-term observations may be explained by metabolic adaptions to lactogenesis, particularly the diversion of glucose by insulin-independent pathways for uptake by the mammary gland [14]. Furthermore, evidence from animal and human studies supports the hypothesis that prolactin, a lactationrelated hormone, plays important regulatory roles in insulin secretion and glucose homeostasis [15-18]. However, very few studies have investigated the biochemical effects of lactation in mothers with gestational diabetes after cessation of lactation [19].

In the present study, we used a targeted metabolomics approach to evaluate metabolomic signatures before and after a glucose challenge in women with prior gestational diabetes. Our objective was to investigate whether lactation for $>3$ months is associated with changes in such signatures up to several years after cessation of lactation.

\section{Methods}

\section{Participants}

We used a dataset of 209 women with a documented diagnosis of gestational diabetes during their most recent pregnancy for the present study. All women underwent an OGTT between 2008 and 2013, either to assess eligibility for the Postpartum Intervention in Women with Gestational Diabetes Using Insulin (PINGUIN) interventional trial (ClinicalTrials.gov NCT01018602; $n=88$ ) or during the clinical study visit of the observational Postpartum Outcomes in Women with Gestational Diabetes and their Offspring (POGO) study $(n=121)$ [20]. The ongoing PINGUIN trial enrolled women with insulin-treated gestational diabetes within 9 months of pregnancy if they ceased lactation, had a non-diabetic glucose tolerance at enrolment and were islet-autoantibody negative. Blood samples obtained from women participating at the PINGUIN screening visit were used to investigate 'shortterm' associations between lactation and metabolomics signatures. The observational POGO study enrolled women with gestational diabetes at 1-10 years postpartum to monitor the development of type 2 diabetes in the women and obesity in the offspring [20]. Blood samples obtained from these women were used to investigate 'long-term' associations between lactation and metabolomics signatures. Participants of the POGO study who were already diagnosed with type 2 diabetes $(n=11)$ or had not yet terminated lactation $(n=1)$ were excluded from the present analyses.

Plasma samples for glucose and insulin assessment and metabolomic profiling were collected at 0,30 and $120 \mathrm{~min}$ during the 75 g OGTT (Dextro O.G.T.; Roche Diagnostics, Mannheim, Germany) from all women. The time from blood collection to centrifugation was $<40 \mathrm{~min}$. Plasma was 
transferred into pre-cooled collection tubes, placed on ice and immediately stored at $-80^{\circ} \mathrm{C}$.

All women attended the clinical study centre of the Institute of Diabetes Research, Helmholtz Zentrum München, Munich, Germany. To ensure standardisation within and between studies, staff were trained to follow standardised procedures with respect to data and sample collection. All participants provided written informed consent. This study was approved by the Ethical Committee of the Technische Universität München, Munich, Germany (PINGUIN study no. 1832/07 and POGO study no. 2937).

\section{Questionnaire and interview data}

Information on lactation duration was collected by interview during the study visits. Women were asked whether they had ever breastfed their child (yes/no) and, if yes, whether the duration of breastfeeding was $>3$ months. Breastfeeding was defined as feeding the newborn either exclusively with breast milk or in addition to other fluids or complementary food. Information on the treatment of gestational diabetes (diet and insulin treatment), BMI at the first antenatal visit and maternal age at delivery were extracted from the participants' obstetric records. The maternal education level was assessed by a questionnaire during the study visit and classified as high (i.e. university qualifications), middle (i.e. secondary school qualifications) or low (i.e. no qualifications or only primary school certificates).

\section{Anthropometric measurements}

During the postpartum study visit, anthropometric data were collected by trained staff using standardised protocols. Maternal weight and height measured in light clothes and without shoes were used to calculate BMI.

\section{Measurement of plasma metabolites}

All plasma samples were measured in two batches comprising four and five plates. Plasma metabolites were measured by electrospray ionisation (ESI) flow injection analysis tandem MS (FIA-MS/MS) and ESI liquid chromatography tandem MS (LC-MS/MS) using AbsoluteIDQ p180 kits (Biocrates Life Sciences, Innsbruck, Austria). This assay can simultaneously quantify 188 metabolites in $10 \mu$ plasma, including 40 acylcarnitines ( $\mathrm{C} x$ : $y$, where $x$ indicates the total number of carbons and $y$ the number of double bonds in the fatty acid side chains), 21 amino acids (19 proteinogenic plus citrulline and ornithine), 21 biogenic amines, total hexoses, 90 glycerophospholipids (14 lysophosphatidylcholines [lysoPCs] and 76 phosphatidylcholines [PCs]), and 15 sphingolipids. All analytical procedures were performed as previously described $[21,22]$. Briefly, $10 \mu$ plasma was pipetted onto a filter which is incorporated into a 96-well sandwich plate and already contains internal standards stable isotope labelled with stable isotope. Amino acids and biogenic amines were derivatised with $5 \%$ phenylisothiocyanate reagent. Metabolites and internal standards were extracted with $5 \mathrm{mmol} / \mathrm{l}$ ammonium acetate in methanol and the solution centrifuged through a filter membrane. For FIA-MS/MS measurements, an aliquot of the resulting solution was diluted in running solvent; for LC-MS/MS, another aliquot was diluted in water.

Quantification of metabolite concentrations and quality assessment were performed with Analyst 1.5 and 1.6 software (for LC-MS/MS) or Met $I D Q$ software (for FIA- and LC-MS/ $\mathrm{MS})$, which is an integral part of the Absolute $I D Q$ p180 kit. The concentration of each metabolite is calculated with reference to appropriate internal standards set by the manufacturer and are reported in $\mu \mathrm{mol} / 1$. The assays were performed at the Genome Analysis Centre of the Helmholtz Zentrum München.

A total of 32 metabolites had a CV of $>25 \%$ in 50 aliquots of a reference plasma sample, which were measured along with the study samples for quality control. These metabolites were therefore excluded from further analyses, leaving a total of 156 metabolites for analysis. In addition to these, we calculated 39 derived traits as sums and ratios of metabolites representing specific metabolite classes (e.g. branched-chain amino acids [BCAAs]) or metabolic processes (e.g. carnitine palmitoyltransferase I [CPT-I] activity via the $[\mathrm{C} 16+\mathrm{C} 18]: \mathrm{C} 0$ ratio; electronic supplementary material [ESM] Table 1) [23].

\section{Measurement of insulin and plasma glucose}

Plasma insulin was analysed by immunoassay using the AIA360 Analyzer (Tosoh Bioscience, Tokyo, Japan). Plasma glucose was measured routinely (Medizet, Munich, Germany) using a photometer (Architect cSystem, Abbott Park, IL, USA). The HOMA-IR index was calculated according to Ekelund et al [24]. The Matsuda-Defronza estimation of insulin sensitivity index (ISI) was calculated according to Vukovic et al [25].

\section{Statistical analysis}

Data collected in the PINGUIN and POGO studies were analysed both together and separately to assess short- and long-term associations, respectively, between lactation and the HOMA-IR, ISI and metabolic profiles. Comparisons between the PINGUIN and POGO studies were performed using the Mann-Whitney $U$ test or $\chi^{2}$ test. For metabolomics analysis, samples were randomly distributed across plates (as described above). If a sample taken at 30 or 120 min during an OGTT was measured on a plate other than the one containing the fasting sample from the same person, then each metabolite in this sample was corrected by the ratio of the mean values for 
the metabolite in the fasting state in both plates to avoid possible batch effects. All metabolite concentrations, sums and ratios were logarithmically transformed to base 10 . Comparisons between women who lactated for $>3$ months and women who lactated for $\leq 3$ months or not at all were performed using profiles obtained at 0,30 and 120 min during the OGTT to specifically address the metabolomic response to a glucose challenge. All comparisons were made using linear regression, with adjustment for age and BMI at the postpartum study visit, time since delivery and maternal education level (high, middle, low); a sensitivity analysis of insulin treatment and potential batch effects was also performed. As 'time since delivery' completely separates participants from the two studies, we did not additionally adjust for 'study type'. Results were corrected for multiple testing by dividing the $5 \%$ level of significance $(\alpha)$ by the 'effective number' of independent tests, which was 67 for our data, according to the method of $\mathrm{Li}$ and Ji [26]. This approach avoids using the overly conservative Bonferroni correction method for correlated data. Therefore, $p$ values of $<0.00075$ (i.e. $\alpha=0.05 / 67$ ) were considered statistically significant.

Gaussian graphical models (GGMs) [27] for single metabolites were calculated based on the full-order partial correlation coefficient $(r)$ values (i.e. pairwise correlations were corrected for all remaining metabolites) for fasting metabolomic samples from both studies combined. GGMs were corrected for age and BMI at the study visit, time since delivery, educational level and lactation by including the respective variables in the partial correlation calculation. The GGM network was generated by drawing a line between any two metabolites for which the partial $r$ value was significantly different from zero (at $\alpha=0.05$ with Bonferroni correction). Associations between metabolite concentrations and lactation for $>3$ months were visualised by colouring network nodes according to the $-\log _{10} p$ value. To ensure a robust GGM with respect to follow-up time, differences between the two subcohorts were assessed using a permutation-based approach (see ESM Text).

Further, we used linear regression models to explore whether HOMA-IR and ISI were associated with lactation duration independently of BMI at the clinic visit, age, time since delivery and educational level or with BCAA levels independently of lactation duration.

Statistical analyses were performed with SAS statistical software (version 9.2, SAS Institute, Cary, NC, USA) and R (version 3.0.0; http://cran.r-project.org) using the GeneNet package [28]. Network colouring was done using Cytoscape (version 2.8, http://chianti.ucsd.edu/Cyto-2_8_0/).

\section{Results}

\section{Characteristics of the participants}

Of the 197 women included in the analysis, 88 (45\%) were assessed shortly after delivery and cessation of lactation (PINGUIN study) and 109 (55\%) were assessed several years postpartum (POGO study; Table 1). As a consequence of the study designs, the time from delivery to postpartum assessment of the metabolomic profiles was significantly shorter for women from the PINGUIN study (median 0.7 years, interquartile range [IQR] $0.4-0.7$ years) than for those from the POGO study (median 6.0 years, IQR 4.1-8.5 years). Accordingly, women from the short-term follow-up cohort were younger at the postpartum study visit compared with women from the long-term follow-up cohort. The proportion of women who reported that they lactated for $>3$ months was
Table 1 Characteristics of the participants

\begin{tabular}{llll}
\hline Characteristic & $\begin{array}{l}\text { Total cohort } \\
(n=197)\end{array}$ & $\begin{array}{l}\text { Short-term (PINGUIN) } \\
(n=88)\end{array}$ & $\begin{array}{l}\text { Long-term (POGO) } \\
(n=109)\end{array}$ \\
\hline $\begin{array}{c}\text { Time from delivery to postpartum } \\
\text { assessment of metabolomics, years }\end{array}$ & $3.6(0.7-6.5)$ & $0.7(0.4-0.7)$ & $6.0(4.1-8.5)^{\mathrm{a}}$ \\
Age, years & $37.5(33.0-41.9)$ & $34.9(30.4-37.8)$ & $40.2(36.0-44.1)^{* * *}$ \\
Insulin treatment during pregnancy & $124(62.9)$ & $88(100.0)$ & $36(33.0)^{* * *}$ \\
Lactation for $>3$ months & $135(68.5)$ & $47(53.4)$ & $88(80.7)^{* * *}$ \\
BMI at follow-up visit, $\mathrm{kg} / \mathrm{m}^{2}$ & $26.3(23.2-30.8)$ & $27.2(23.8-31.4)$ & $25.6(22.6-30.2)$ \\
Obese $\left(\mathrm{BMI}>30 \mathrm{~kg} / \mathrm{m}^{2}\right)$ & $61(31.0)$ & $32(36.4)$ & $29(26.7)$ \\
$\begin{array}{l}\Delta \mathrm{BMI} \\
\mathrm{b}\end{array} \mathrm{kg} / \mathrm{m}^{2}$ & $1.0(-0.4-2.3)$ & $1.0(0-2.2)$ & $1.0(-0.7-2.3)$ \\
Higher educational level & $92(46.7)$ & $41(46.6)$ & $51(46.8)$ \\
$\quad(\mathrm{vs}$ middle and lower) & & & \\
\hline
\end{tabular}

Data are the median (IQR) or $n(\%)$

${ }^{a}$ This comparison was not tested for significance because the difference is entirely due to the different study designs

${ }^{\mathrm{b}} \Delta \mathrm{BMI}=\mathrm{BMI}$ at follow-up visit $-\mathrm{BMI}$ in early pregnancy

$* * * p<0.001$ 
Fig. 1 Metabolite concentrations $($ mean $\pm 1.96 \times \mathrm{SE})$ for $(\mathbf{a}-\mathbf{c})$ total lyso-PC/total $\mathrm{PC}$ ratios, $(\mathbf{d}-\mathbf{f})$ BCAA levels and (g-i) leucine levels at 0 (fasting) and 30 and 120 min during OGTTs in women who lactated for $>3$ months or for $\leq 3$ months or not at all. Results are shown for $(\mathbf{a}, \mathbf{d}, \mathbf{g})$ both cohorts combined and for $(\mathbf{b}, \mathbf{e}, \mathbf{h})$ participants in the short-term follow-up (PINGUIN) and (c, f, i) long-term follow-up (POGO) studies. $p$ values were derived from comparisons of the two lactation groups in linear regression models, with adjustment for maternal age and BMI at the postpartum study visit, time since delivery and maternal education. ${ }^{\dagger}$ Significant associations (i.e. $\alpha=0.05 / 67$, $p<0.00075)$. Black circles, lactation for $\leq 3$ months; white circles, lactation for $>3$ months
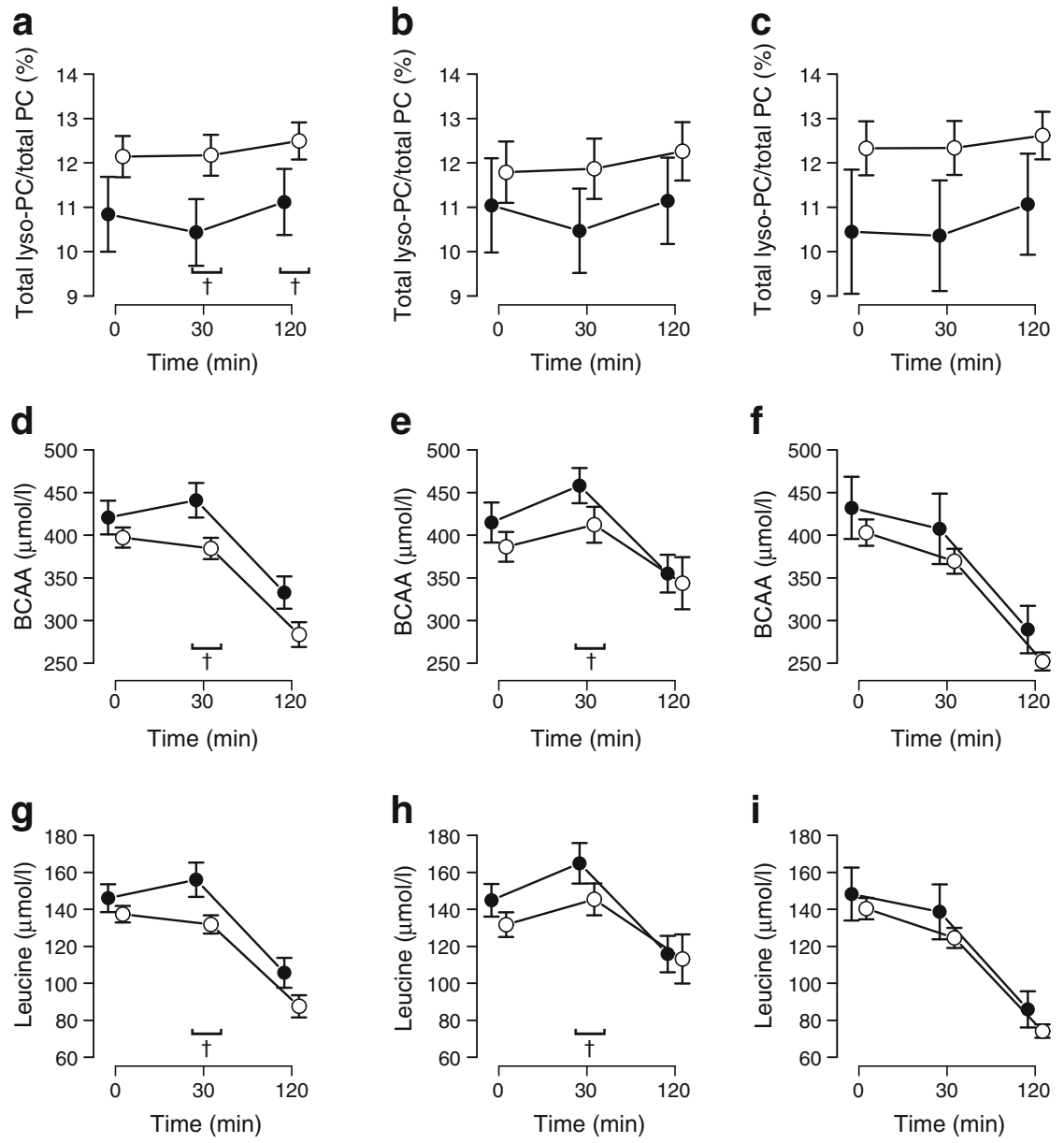

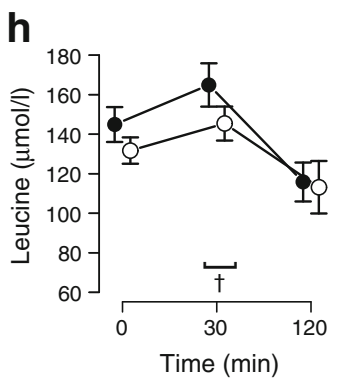

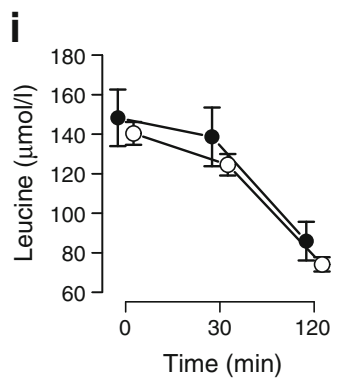

greater in the long-term than in the short-term follow-up cohort $(81 \%$ vs $53 \%, p<0.0001)$. Postpartum BMI, the prevalence of obesity and educational level were similar for women in both studies. Although women in the short-term follow-up cohort were assessed nearer to delivery, their postpartum change in $\mathrm{BMI}(\triangle \mathrm{BMI}=[\mathrm{BMI}$ at follow-up visit $]-[\mathrm{BMI}$ in early pregnancy]) was comparable with that observed in the long-term follow-up cohort.

\section{Metabolomic signatures of lactation for $>\mathbf{3}$ months}

Linear regression analysis of the total cohort revealed that lactation for $>3$ months was significantly associated (all $p<0.00075$ ) with higher total lyso-PC/total $\mathrm{PC}$ ratios at 30 and 120 min during the OGTT after adjusting for BMI, age, time since delivery and educational level. Additionally, in the subset with short-term follow-up, lactation for $>3$ months was associated with a lower concentration of leucine (a BCAA) and of total BCAAs (i.e. valine, leucine and isoleucine) at $30 \mathrm{~min}$ of the OGTT (all $p<0.00075$; Fig. 1 and ESM Table 2). These results were very similar when the analyses were additionally adjusted for insulin treatment and potential batch effects (data not shown).

In the next step, we calculated a GGM based on partial $r$ values for fasting metabolomic samples of the
Table 2 Association of lactation duration with insulin sensitivity and insulin resistance $\mathrm{e}^{\mathrm{a}, \mathrm{b}}$

\begin{tabular}{llll}
\hline Measure & $\begin{array}{l}\text { Total cohort } \\
(n=197)\end{array}$ & $\begin{array}{l}\text { Short-term follow-up } \\
\text { (PINGUIN) }(n=88)\end{array}$ & $\begin{array}{l}\text { Long-term follow-up } \\
\text { (POGO) }(n=109)\end{array}$ \\
\hline ISI Matsuda-Defronza & $-1.34(-3.90,1.21)$ & $-0.34(-2.44,1.76)$ & $-2.73(-7.45,1.99)$ \\
HOMA-IR & $-0.34(-0.76,0.09)$ & $-0.63(-0.76,0.64)$ & $-0.53(-1.15,0.09)$ \\
\hline
\end{tabular}

Data are the $\beta$ coefficient $(95 \% \mathrm{CI})$

${ }^{a}$ Lactation for $>3$ months compared with lactation for $\leq 3$ months

${ }^{\mathrm{b}}$ Adjusted for BMI at the postpartum study visit, age, time since delivery and educational level 

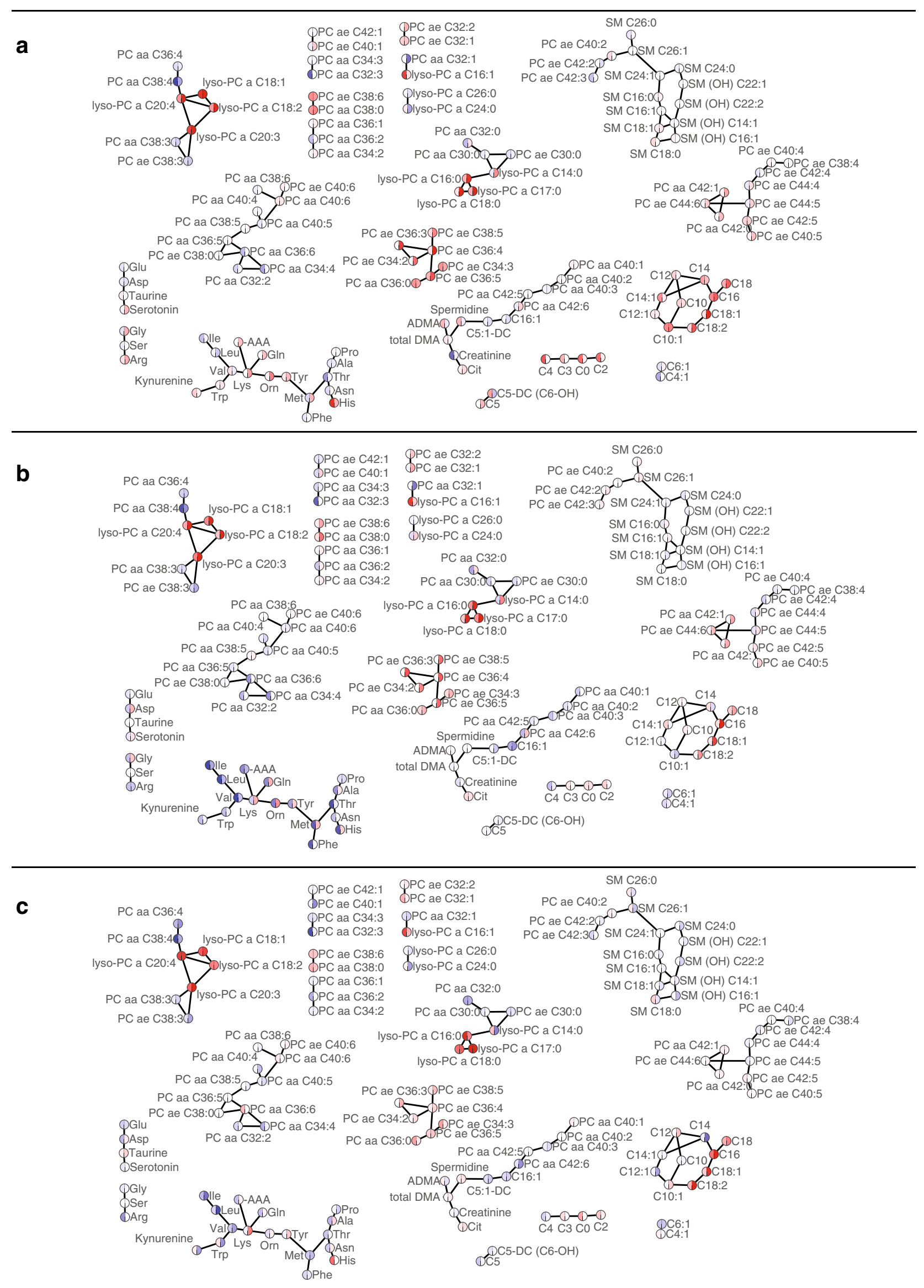
4 Fig. 2 Gaussian graphical model of all plasma metabolites measured in the study, illustrating the correlation strength and effects mediated by lactation separated by follow-up time. Each node represents a single metabolite. Node colour represents the strength of association between lactation for $>3$ months and metabolite concentrations (blue, lower concentration in women who lactated for $>3$ months; red, higher concentration in women who lactated for $>3$ months) at (a) 0 min (i.e. fasting), (b) 30 min during the OGTT and (c) 120 min during the OGTT. For each node, the left part represents associations in the short-term follow-up group (PINGUIN) and the right part represents associations in the long-term follow-up group (POGO). PC aa, diacylphosphatidylcholine; PC ae, acyl-alkyl-phosphatidylcholine

total cohort to visualise networks between single metabolites and their association with lactation for $>3$ months (Fig. 2). Within the network, we identified several clusters of phospholipids, including lyso-PCs, diacyl-PCs and acyl-alkyl-PCs that are affected by lactation for $>3$ months. The network showed higher concentrations of several lyso-PCs, lower concentrations of several diacyl-PCs and, to a lesser extent, lower concentrations of acyl-alkyl-PCs in women lactating for $>3$ months, although none of the differences in single metabolites had been significant at $p<0.00075$ in linear regression analyses. The diacyl-PCs and lyso-PCs that differed between lactation for $>3$ months and lactation for $\leq 3$ months were connected within smaller clusters of glycerophospholipids. Lactation-associated differences in glycerophospholipid concentrations were observed in the fasting state (Fig. 2a) and at 30 and 120 min (Fig. 2b,c) during the OGTT. These findings were similar with respect to followup duration after delivery, although lactation for $>3$ months seemed to be predominantly associated with lower concentrations of several diacyl-PCs in the short-term follow-up group and higher concentrations of lyso-PCs in the long-term followup group. The network analysis further indicated strongly correlations among levels of the BCAAs leucine, isoleucine and valine, and these were lower in women lactating for $>3$ months. Metabolite networks were not significantly different after calculating separate GGMs for women lactating for $\leq 3$ months and women lactating for $>3$ months (ESM Text). Differences in networks between the different follow-up groups were negligible (ESM Text).

\section{Associations of the ISI and HOMA-IR with BCAA levels and lactation duration}

The ISI was negatively associated $(\beta=-1.96$ [95\% CI $-3.52,-0.40]$ per $100 \mu \mathrm{mol} / 1$ increase in BCAA) and the HOMA-IR was positively associated $(\beta=0.68[95 \% \mathrm{CI}$ $0.37,0.98]$ per $100 \mu \mathrm{mol} / 1$ increase in BCAA) with BCAA levels after adjustment for lactation duration. Lactation for $>3$ months was not associated with the ISI and HOMA-IR after adjusting for BMI at the clinic visit, age, time since delivery and educational level in the total cohort nor in separate analyses of the short-term and longterm follow-up cohorts (Table 2).

\section{Discussion}

In women with prior gestational diabetes, lactation for $>3$ months was significantly associated with a higher total lyso-PC/total PC ratio at 30 and $120 \mathrm{~min}$ during an OGTT within 3.6 years postpartum, and also with lower leucine and lower total BCAA (valine, leucine, isoleucine) concentrations at $30 \mathrm{~min}$ within 0.7 years postpartum. Lactation for $>3$ months was not associated with insulin sensitivity (ISI) or insulin resistance (HOMA-IR).

Glycerophospholipid and BCAA levels are reported to be related to insulin resistance, type 2 diabetes and obesity [29-33]. Moreover, population-based studies of previously healthy adults revealed that low lyso-PC C18:2 concentrations were associated with an increased risk of pre-type 2 diabetes and type 2 diabetes [30, 34], while high concentrations of several diacyl-PCs (C32:1, C36:1, C38:3 and C40:5) were associated with a risk of type 2 diabetes independent of dietary, lifestyle and anthropometric factors. In our study, the total lyso-PC/total PC ratio was significantly increased in women who had lactated for $>3$ months as a consequence of higher concentrations of several lyso-PCs and lower concentrations of several diacyl-PCs in these women. However, these associations were not statistically significant at the single metabolite level after correction for multiple testing.

In our study, lactation for $>3$ months was associated with lower leucine concentrations and total BCAA concentrations at $30 \mathrm{~min}$ of the OGTT up to 0.7 years postpartum. A decrease in plasma leucine concentrations after a glucose challenge has been reported in healthy adults [35]. In contrast, increased concentrations of (iso)leucine have been reported after a glucose challenge and standardised meal challenge in individuals with insulin resistance and in patients with type 2 diabetes [33, 36]. Furthermore, it was recently reported that pregnant women with high fasting plasma glucose concentrations also had high fasting concentrations of several amino acids, including leucine [37]. Higher fasting concentrations of BCAAs were also found in individuals with type 2 diabetes and insulin resistance [34, 38-40]. These findings indicate that alterations in BCAA metabolism are associated with gestational diabetes, insulin resistance and type 2 diabetes. Clinical intervention studies have also reported that decreased BCAA concentrations following an intervention (e.g. gastric bypass surgery or medical therapy) correlated with improvements in glucose homeostasis [41, 42].

One hypothesis to explain how increased BCAA concentrations influence the risk of type 2 diabetes is that increased 
circulating concentrations of BCAAs interfere with fatty acid oxidation, and thus decrease insulin sensitivity [40]. Recent studies propose that the intermediate metabolites of BCAA catabolism are associated with insulin sensitivity and type 2 diabetes and not the BCAA concentrations themselves [38, 39]. Indeed, in our study BCAA concentrations were associated with ISI and HOMA-IR and lactation for $\leq 3$ months was associated with increased BCAA concentrations early after cessation of lactation. However, lactation for $\leq 3$ months was not associated with decreased insulin sensitivity, suggesting that the mechanism responsible for diabetes protection by prolonged lactation may be independent of insulin sensitivity and that other mechanisms, possibly involving BCAAs, may be more relevant.

Metabolites identified in previous metabolomics studies as being associated with a risk of type 2 diabetes serve as biomarkers for disturbed metabolic pathways in the early pathogenesis of type 2 diabetes [34]. We argue that lactation for $>3$ months may protect women with gestational diabetes from later developing type 2 diabetes by either 'resetting' these disturbed pathways or preventing further disturbances in some pathways, specifically the phospholipid and BCAA pathways. Moreover, we hypothesise that lactation-associated changes in metabolomic signatures, particularly associated with PC metabolism, may persist for several years postweaning because the observed associations were independent of the time from delivery to the postpartum assessment. However, the mechanisms through which lactation affects these metabolic pathways are still unclear. Data from animal and human studies suggest that lactation helps to reset maternal metabolism after pregnancy by mobilising accumulated fat stores and reducing adiposity [8]. However, it is notable that changes in the metabolomic signatures of women who lactated for $>3$ months were not attenuated after adjusting for maternal BMI.

To our knowledge, this was the first study to use a targeted metabolomics approach to quantify a broad spectrum of lipids and amino acids and to identify lactation-associated biochemical changes in plasma samples collected from women with prior gestational diabetes. The primary strength of our study is the analysis of metabolomic signatures during an OGTT in a well-characterised population of 197 women who were diagnosed with gestational diabetes using a standardised OGTT. In addition, we had the opportunity to evaluate the short- and long-term effects of prolonged lactation by including women who participated in this study either soon or several years after delivery.

A potential limitation of the study is that the duration of lactation was retrospectively determined by interviews done at a median of 3.6 years postpartum. Some studies suggest that recall bias of lactation behaviour tends to increase with the time elapsed since weaning: women who lactated for a short period are more likely to exaggerate the duration of lactation, while the opposite was observed for women who lactated for a long period [43-45]. Moreover, lactating women tended to be more health conscious, with better physical activity or dietary behaviours [45], so we cannot exclude the possibility that different lifestyle factors may have affected our results. Notably, however, lifestyle behaviours did not affect the association between lactation and long-term protection against type 2 diabetes in a recent population-based study [10]. Since all women were white, our results may not be transferable to other racial or ethnic groups. All members of the subset of women with short-term follow-up had insulin-treated gestational diabetes and were therefore at a very high risk of developing type 2 diabetes; their risk profile differs markedly from that of women in the long-term follow-up group. On the other hand, associations between lactation for $>3$ months and metabolite levels did not change after adjusting for insulin treatment. Based on a significance level of 0.00075 and a desired statistical power of $80 \%$, our setting enabled us to identify group differences of 0.66 SDs or more for each metabolite. Thus, we cannot preclude the possibility that we failed to detect smaller associations for other metabolites.

In conclusion, our study provides evidence that lactation for $>3$ months may alter the metabolomic signature previously reported to be associated with a decreased risk of type 2 diabetes, and that these alterations persist for several years postweaning. Therefore, the lower risk of type 2 diabetes conferred by lactation may be mediated by several metabolic pathways involved in early disease pathogenesis.

Acknowledgements We thank M. Bunk and H. Jaschinski, Institute for Diabetes Research, Helmholtz Zentrum München, Germany, for sample collection at study site visits and for performing the OGTTs. We also thank J. Scarpa and K. Faschinger, Genome Analysis Center, Metabolomics Core Facility, Helmholtz Zentrum München, Germany, for performing the metabolomic profiling.

Funding The POGO study is funded by the Institute of Diabetes Research, Helmholtz Zentrum München, Neuherberg, Germany, and by the German Center for Diabetes Research (DZD). The PINGUIN study is partly funded by a grant from the German Federal Ministry of Education and Research (BMBF) to Forschergruppe Diabetes e.V. and directly by Forschergruppe Diabetes e.V. This study was supported in part by a grant from the BMBF to the DZD and by the Helmholtz Cross-Program Initiative Personalized Medicine iMED, the European Union's Horizon 2020 funded DynaHEALTH research project (no. 633595), and project grants from the German Diabetes Association and the Helmholtz International Research Group (Helmholtz HIRG-0018).

Duality of interest statement The authors declare that there is no duality of interest associated with this manuscript.

Contribution statement A-GZ designed the study and is the principal investigator of the study; AH, DM, DW, MH, MR and SHi contributed to data acquisition; $\mathrm{AB}$ performed data analysis, with contributions from $\mathrm{SHu}, \mathrm{A}-\mathrm{GZ}$ and $\mathrm{DM}$; CP, WR-M and JA performed the metabolomics analysis; AK, JK and FS performed the network analysis; FT contributed to metabolomics data interpretation; DM, GK and SHu contributed to data interpretation; DM drafted the article; AB, AK, CP, FS, GK, JA, $\mathrm{JK}$ and WR-M critically reviewed the manuscript; $\mathrm{AB}, \mathrm{A}-\mathrm{GZ}, \mathrm{CP}, \mathrm{FS}$, FT, GK, JA, JK, SHu and WR-M contributed to the final draft of the 
manuscript. SHu is the guarantor of this work and, as such, had full access to all the data in the study and takes responsibility for the integrity of the data and the accuracy of data analysis. All authors critically revised the article for important intellectual content and gave final approval of the version to be published.

\section{References}

1. Ip S, Chung M, Raman G et al. (2007) Breastfeeding and maternal and infant health outcomes in developed countries. Evid Rep Technol Assess 153:1-186

2. Harder T, Bergmann R, Kallischnigg G, Plagemann A (2005) Duration of breastfeeding and risk of overweight: a meta-analysis. Am J Epidemiol 162:397-403

3. Ziegler AG, Wallner M, Kaiser I et al (2012) Long-term protective effect of lactation on the development of type 2 diabetes in women with recent gestational diabetes mellitus. Diabetes 61:3167-3171

4. Much D, Beyerlein A, Roßbauer M, Hummel S, Ziegler A (2014) Beneficial effects of breastfeeding in women with gestational diabetes mellitus. Mol Metab 3:284-292

5. Amir LH, Donath S (2007) A systematic review of maternal obesity and breastfeeding intention, initiation and duration. BMC Pregnancy Childbirth 7:9

6. Hilson JA, Rasmussen KM, Kjolhede CL (2006) Excessive weight gain during pregnancy is associated with earlier termination of breast-feeding among White women. J Nutr 136:140-146

7. Gunderson EP (2014) Impact of breastfeeding on maternal metabolism: implications for women with gestational diabetes. Curr Diab Rep 14:460

8. Stuebe AM, Rich-Edwards JW (2009) The reset hypothesis: lactation and maternal metabolism. Am J Perinatol 26:81-88

9. Stuebe AM, Rich-Edwards JW, Willett WC, Manson JE, Michels KB (2005) Duration of lactation and incidence of type 2 diabetes. JAMA 294:2601-2610

10. Jager S, Jacobs S, Kroger J et al (2014) Breast-feeding and maternal risk of type 2 diabetes: a prospective study and meta-analysis. Diabetologia 57:1355-1365

11. Tigas S, Sunehag A, Haymond MW (2002) Metabolic adaptation to feeding and fasting during lactation in humans. J Clin Endocrinol Metab 87:302-307

12. Lenz S, Kuhl C, Hornnes PJ, Hagen C (1981) Influence of lactation on oral glucose tolerance in the puerperium. Acta Endocrinol 98: $428-431$

13. Knopp RH, Walden CE, Wahl PW et al (1985) Effect of postpartum lactation on lipoprotein lipids and apoproteins. J Clin Endocrinol Metab 60:542-547

14. Butte NF, Hopkinson JM, Mehta N, Moon JK, Smith EO (1999) Adjustments in energy expenditure and substrate utilization during late pregnancy and lactation. Am J Clin Nutr 69:299-307

15. Park S, Kang S, Lee HW, Ko BS (2012) Central prolactin modulates insulin sensitivity and insulin secretion in diabetic rats. Neuroendocrinology 95:332-343

16. Crepaldi SC, Carneiro EM, Boschero AC (1997) Long-term effect of prolactin treatment on glucose-induced insulin secretion in cultured neonatal rat islets. Horm Metab Res 29:220-224

17. Wang T, Lu J, Xu Y et al (2013) Circulating prolactin associates with diabetes and impaired glucose regulation: a population-based study. Diabetes Care 36:1974-1980

18. Ramos-Roman MA (2011) Prolactin and lactation as modifiers of diabetes risk in gestational diabetes. Horm Metab Res 43:593-600

19. Gunderson EP (2013) The role of lactation in GDM women. Clin Obstet Gynecol 56:844-852
20. Hummel S, Much D, Rossbauer M, Ziegler AG, Beyerlein A (2013) Postpartum outcomes in women with gestational diabetes and their offspring: POGO study design and first-year results. Rev Diabet Stud 10:49-57

21. Zukunft S, Sorgenfrei M, Prehn C, Möller G, Adamski J (2013) Targeted metabolomics of dried blood spot extracts. Chromatographia: 1-11

22. Römisch-Margl W, Prehn C, Bogumil R, Röhring C, Suhre K, Adamski J (2012) Procedure for tissue sample preparation and metabolite extraction for high-throughput targeted metabolomics. Metabolomics 8:133-142

23. Fingerhut R, Roschinger W, Muntau AC et al (2001) Hepatic carnitine palmitoyltransferase I deficiency: acylcarnitine profiles in blood spots are highly specific. Clin Chem 47:1763-1768

24. Ekelund M, Shaat N, Almgren P, Groop L, Berntorp K (2010) Prediction of postpartum diabetes in women with gestational diabetes mellitus. Diabetologia 53:452-457

25. Vukovic R, Mitrovic K, Milenkovic T et al (2013) Insulin-sensitive obese children display a favorable metabolic profile. Eur J Pediatr 172:201-206

26. Li J, Ji L (2005) Adjusting multiple testing in multilocus analyses using the eigenvalues of a correlation matrix. Heredity 95:221-227

27. Krumsiek J, Suhre K, Illig T, Adamski J, Theis F (2011) Gaussian graphical modeling reconstructs pathway reactions from highthroughput metabolomics data. BMC Syst Biol 5:21

28. Krumsiek J, Suhre K, Evans AM et al (2012) Mining the unknown: a systems approach to metabolite identification combining genetic and metabolic information. PLoS Genet 8:e1003005

29. Wang TJ, Larson MG, Vasan RS et al (2011) Metabolite profiles and the risk of developing diabetes. Nat Med 17:448-453

30. Wang-Sattler R, Yu Z, Herder C et al (2012) Novel biomarkers for pre-diabetes identified by metabolomics. Mol Syst Biol 8:615

31. Ferrannini E, Natali A, Camastra S et al (2013) Early metabolic markers of the development of dysglycemia and type 2 diabetes and their physiological significance. Diabetes 62:1730-1737

32. Bentley-Lewis R, Xiong G, Lee H, Yang A, Huynh J, Kim C (2014) Metabolomic analysis reveals amino-acid responses to an oral glucose tolerance test in women with prior history of gestational diabetes mellitus. J Clin Transl Endocrinol 1:38-43

33. Mook-Kanamori DO, de Mutsert R, Rensen PC et al (2015) Type 2 diabetes is associated with postprandial amino acid measures. Arch Biochem Biophys 589:138-144

34. Floegel A, Stefan N, Yu Z et al (2013) Identification of serum metabolites associated with risk of type 2 diabetes using a targeted metabolomic approach. Diabetes 62:639-648

35. Shaham O, Wei R, Wang TJ et al (2008) Metabolic profiling of the human response to a glucose challenge reveals distinct axes of insulin sensitivity. Mol Syst Biol 4:214

36. Ho JE, Larson MG, Vasan RS et al (2013) Metabolite profiles during oral glucose challenge. Diabetes 62:2689-2698

37. Scholtens DM, Muehlbauer MJ, Daya NR et al (2014) Metabolomics reveals broad-scale metabolic perturbations in hyperglycemic mothers during pregnancy. Diabetes Care 37:158-166

38. Newgard CB, An J, Bain JR et al (2009) A branched-chain amino acid-related metabolic signature that differentiates obese and lean humans and contributes to insulin resistance. Cell Metab 9:311-326

39. Menni C, Fauman E, Erte I et al (2013) Biomarkers for type 2 diabetes and impaired fasting glucose using a nontargeted metabolomics approach. Diabetes 62:4270-4276

40. Newgard CB (2012) Interplay between lipids and branched-chain amino acids in development of insulin resistance. Cell Metab 15: 606-614

41. Lips MA, van Klinken JB, van Harmelen V et al (2014) Roux-en-Y gastric bypass surgery, but not calorie restriction, reduces plasma branched-chain amino acids in obese women independent of weight loss or the presence of type 2 diabetes. Diabetes Care 37:3150-3156 
42. Walford GA, Davis J, Warner AS et al (2013) Branched chain and aromatic amino acids change acutely following two medical therapies for type 2 diabetes mellitus. Metab Clin Exp 62:1772-1778

43. Promislow JH, Gladen BC, Sandler DP (2005) Maternal recall of breastfeeding duration by elderly women. Am J Epidemiol 161: 289-296
44. Huttly SR, Barros FC, Victora CG, Beria JU, Vaughan JP (1990) Do mothers overestimate breast feeding duration? An example of recall bias from a study in southern Brazil. Am J Epidemiol 132: $572-575$

45. Horta BL, Victora CG (2013) Long-term effects of breastfeeding: a systematic review. World Health Organization, Geneva 